\title{
A Dependable Communication Network for e-Textiles
}

\author{
Nenggan Zheng, Zhaohui Wu, Lei Chen, Yanmiao Zhou, and Qijia Wang \\ College of Computer Science and Technology, \\ Zhejiang University, 310027, Hangzhou, P.R. China \\ \{zng, wzh, leisteven, yanmiaozhou\}@zju.edu.cn, \\ gotowqje163. com
}

\begin{abstract}
Due to high frequent wear and tear or other faults in use, it is important to implement a fault-tolerant communicating network for e-textiles that can be easily woven into a fabric. In this paper, we introduce token buses to connect the nodes, instead of the single rings in the original e-textile token grid. The topology of the new network is described. And we also discuss the media access control protocols and the reliable operations. Simulation results show that the new e-textile communication network can improve the ability of e-textile applications to tolerate faults and provide the communicating services of less delay.
\end{abstract}

\section{Introduction}

Electronic textiles (e-textiles), also called smart fabrics, are emerging new computing substrates, which combine the advantages and abilities of electronic modules and textiles into one [1]. People in this research field wear off-the-shelf electrical components such as microprocessors, sensors and conductive strands into traditional clothing materials. Potential applications for e-textiles include medical monitoring, military uniforms and ambient computing devices [2]. And several prototypes based on etextiles are presented in the papers and websites available [1, 3-5].

When the fabrics are tailored as a wearable garment or when the applications are in use, tear and wear are highly frequent, which potentially introduce link or node failures into the communication network. The failures will lead to the destruction of a local part in the communication network and even the collapse of the whole distributed system. Consequently, it is important to implement a fault-tolerant communicating network for e-textiles that can be easily woven into fabrics. The physical layout of the communication network should be run in one of two perpendicular directions, which is determined by the weaving process. Z. Nakad et al. modify the Token Grid Network (as shown in Fig. 1(a)) of [6] and propose the e-textile token grid that has an added "transverse" dimension between two token grids [7].

With the added "transverse" dimension, the e-textile token grid has the capacity to support large numbers of nodes, thus not limited by the width direction of the fabric size. By interconnecting the nodes in different grids, the added dimension can decrease the number of the nodes in a ring and directly reduce the delay time to wait for the tokens. Accounting for the demand of routing around the dormant or disabled nodes, as shown in Fig. 1, communication protocols implement the "Wrong Routing" technology [8]. 


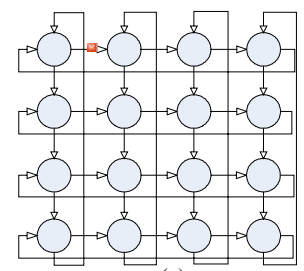

(a)

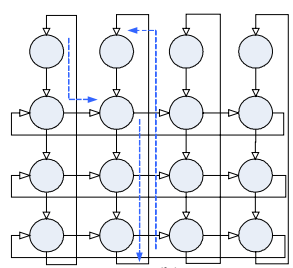

(b)
Wrong Route

Fig. 1. (a) A link fault on the top Row ring (The red square in the figure represents a link fault.) (b) The communication on the top Row ring breaks down. "Wrong Routing" technology is used to route the data packet between the nodes on this Row ring with a link fault.

The e-textile token grid provides a communicating scheme with considerable faulttolerant ability for e-textiles in dynamic and harsh environments. However, Z. Nakad's e-textile token grid network can not tolerate faults simultaneously present on every ring. The e-textile token grid network offers the fault-tolerant operations for the communication services, but the distributed system still breaks down in the presence of the simultaneous faults in every ring (as illustrated in Fig. 2). The reason for this result lies in the fact that the nodes are interconnected by single rings. A link failure is sufficient to block off the data traffic on a single ring. And a node fault will stop the communications on the two perpendicular rings converging on this node, regarded as two output link faults of the node. For the case (simultaneous faults on every row or column rings) is potentially high frequent in the manufacturing process or in use, the communication network for e-textiles should have the ability to recover from the corrupt state. In this paper, we aim to introduce a token bus to connect the nodes, instead of the single ring in the original token grid and construct the e-textile grid network with token bus (EGNTB). With the token bus and its fault-tolerant media access control protocol, faults can only affect the nodes with errors and have result in less delay time.

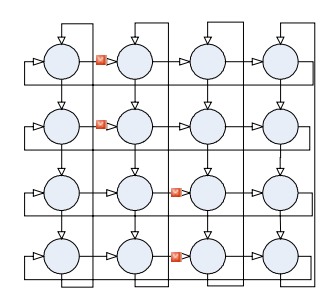

(a)

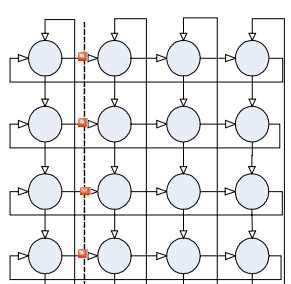

(b)
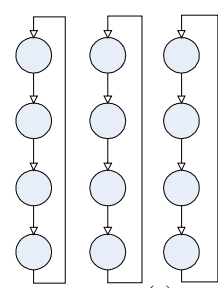

(c)

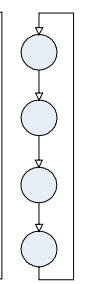

Fig. 2. Serious faults resulting in the destructions of the communication on the whole token grid. (c) is the result of (a) or (b). Note that the faults are indicated as red squares in the figure.

The remainder of this paper is organized as follows: In section 2, the topology of the e-textile token grid with specific token bus is illustrated and the operations of the networks in presence of tears are also discussed. Next, section 3 evaluates the throughput of the new network in the case of uniform load. Simulation experiments 
are also conducted to obtain the time delay of both the EGNTB and the e-textile original token grid with the failures. Finally, we conclude the paper in section 4 .

\section{2 e-Textile Grid Network with Token Bus}

The e-textiles grid network with token bus (EGNTB) is introduced in this section. The modification to the topology is discussed. And the fault-tolerant media control access protocols are introduced in two subsections. The basic operations are described firstly in subsection 2.1, and then we will discuss the operations on the new network with the serious faults.

\subsection{Topology and Basic Protocols of the EGNTB}

The EGNTB (e-textile grid network with token bus) is a two-dimensional network structure arranged in $\mathrm{M}$ rows and $\mathrm{N}$ columns. With token buses in row and column, each node is connected to a row bus and a column bus. Fig. 3(a) depicts an example of four columns and four rows. The address of every node is denoted as (RowID, ColumnID). Each token bus is referred by its respective row or column number. For instance, the top row token bus is named as row-token-bus 1 (RTB1) while the leftmost column token bus is identified as column-token-bus 1 (CTB1). For each node is connected onto two token buses, two pairs of transmitters and receivers are necessary to implement the EGNTB. Thanks to the bus topology of the new network, the "transverse" dimension can be easily implemented by connecting the corresponding row/column token bus of the grids in two perpendicular directions. Additional hardware interfaces are saved.

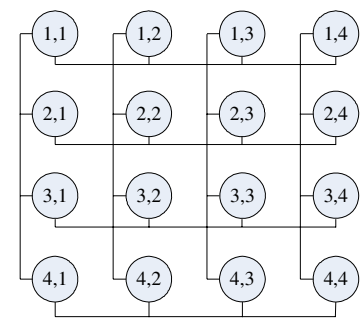

(a)

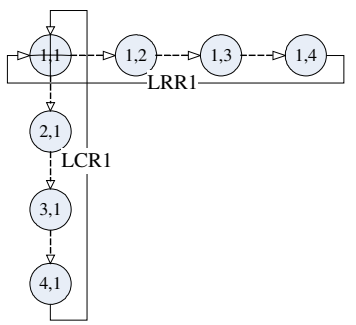

(b)

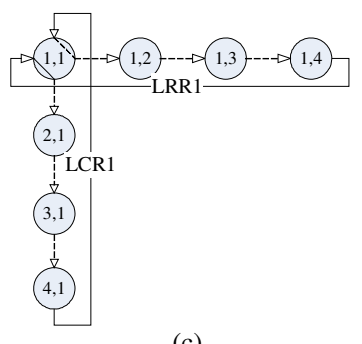

(c)

Fig. 3. (a) The e-textile grid network with specific token bus (EGNTB) (b) A logical row ring (LRR1) and a logical column ring (LCR1) converging on node $(1,1)$ while the node is in DR configuration (c) Node $(1,1)$ in SR configuration

The specific MAC protocol controls the tokens that are passed from a node to its active subsequent one on the buses. Different from IEEE 802.4 standard for LAN, the nodes on a row/column token bus of the EGNTB form a logical token ring without token competition. As shown in Fig. 3(b), two logical token rings converging on Node $(1,1)$ which are named as logical-row-ring1 (LRR1) and logical-column-ring1 (LCR1) respectively. To simplify the description of the network, we assume that all of the logical row rings have the directions pointing to the right and all of the logical 
column rings have the directions pointing to the bottom of the figure. Each node has the same chance to grasp the token and keep it for sending data packets on the logical token ring. The basic protocols assign every node has the same period that can be adapted to the real-life requirements of the applications. As an advantage of the token rings, except for the usual few bits of station latency, other bits of communicating buffer are not required in the node of the EGNTB.

The token circulated on every logical ring to keep the information of the network and control the access chance to the communication channel. Node set on the logical ring is preserved in the nodes by a bitmap of variable length. The bits can be mapped to the ID table for routing the packets. Dormant or disabled nodes are considered by the inactive bits that are encapsulated in the token. Because the EGNTB implement a logical topology of the original token rings, the basic operation protocol of EGNTB are similar to the original e-textile token grid networks in [6] and [7]. The basic token grid operation protocol is described formally in [6]. The nodes in the network have two configurations: the single-ring connection (SR) and the double-ring (DR) connection (shown in Fig. 3(c) and (b)). For a node with the DR configuration, the row ring and the column ring converging at this node are separate. While a node is in the merged configuration, the two rings are merged into a new token ring. If an active node wants to send a packet to another node on the same row or the same column, the node should wait for the corresponding ring token and seize it when the token arrives. And while the destination of the data packet on the different row and column, that is, in the case of the source node $\left(R_{1}, C_{1}\right)$ and the destination node $\left(R_{2}, C_{2}\right)$, the operation protocol will make the node $\left(R_{1}, C_{2}\right)$ or the node $\left(R_{2}, C_{1}\right)$ is in the SR configuration to connect the source node with the destination one into a ring. For example, if node (4, 1) sends a packet to node $(1,2)$, the operation protocol can require node $(1,1)$ or node $(4,2)$ be in the SR configuration to form a new merged ring and carry the data packet. The protocol also supports the inter communication between grids by supporting the address of the different grid IDs and buffers of appropriate sizes.

\subsection{Fault Tolerant Operations}

As discussed in the section 1, there are still two potential cases of the faults that can disable the whole network, though the existing e-textiles networks provide considerable robust operations for applications. By reason of the high frequent abrasions or for the need of manufacturing e-textile applications, the simultaneous link failures on the row rings or the column rings often happen. Firstly, a tear across the width of a fabric will sever the token grid into two parts as shown in Fig. 2(b). Both the parts will only own the column rings and the full connectivity in every part is lost. The remaining nodes can not communicate with any node on different columns or rings. Secondly, the power-efficient characteristics of e-textiles require the power consuming nodes to enter into a sleep state to conserve power energy. A node in the failure state or in the dormant state is equivalent to two link failures. That is, a node failure or dormant is treated as the link failures of its two communication outputs. In the case of every ring with a node failure or dormant, the communication of original token grid network will be completely broken out. For the cases discussed above, the existing token grid network for e-textiles needs to enhance its fault-tolerant ability to achieve more robust operations. 
The difference between Z. Nakad's e-textile token grid network and EGNTB lies in that each node in latter is connected by logical token rings based on the specific MAC protocol. The new network benefits from the token bus and the protocol, thus owning the inherent high reliability. This is the point of how we enhance the fault-tolerant ability of the EGNTB and make it more suitable for e-textiles applications. The error detection algorithm with delay-time counters is used for checking the link or node faults [4] [8]. When a fault is found by the time counters in the nodes, the protocol update the inactive bits in the token. Both the node fault and the bus fault are detected and treated as two link failures on the logical token ring. For a node fault, as shown in Fig. 4(a), the fault is regarded as its input link failure and output link failure in Fig. 4(b). While in the presence of the fault on the token bus, the fault will partition the node set on the bus into two subsets, which is illustrated in Fig. 5(a) and (b). Note that the faults are indicated as red squares in the figure.
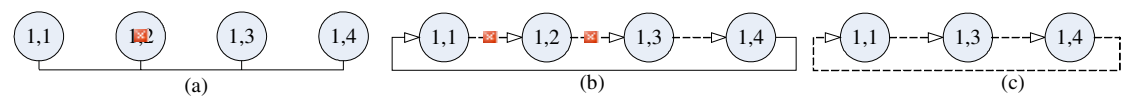

Fig. 4. Node fault: (a) Node $(1,2)$ in failure state (b) Two link faults equivalent on the logical token ring (c) The logical ring after fault-tolerant operations is performed
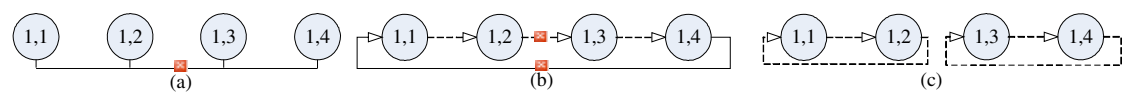

Fig. 5. A fault on the token bus: (a) A fault on the bus between node $(1,2)$ and node $(1,3)$ (b) Two link faults equivalent on the logical token ring which will partition the former ring into two sub rings (c) The result of fault-tolerant operations

When a node faults are checked, the inactive bits in the token are updated to reflect the dynamic variation on the bus and the failure node is eliminated from the logical ring. The number of the nodes on the logical token ring decreases by one, as shown in Fig. 4 (c). The drop in number of the nodes, however, leads to less time delay in transferring the data packet.

In the case of a fault on the token bus, the fault cuts the bus into two parts. Network information including inactive bits and the node set of the logical ring is updated by an error-broadcasting token. The node set of the logical ring is divided into two subsets by the fault. And for those nodes in the other subset, the associated inactive bits are set up to present that the node is not connected onto the logical ring of the current subset. Consequently, the former logical ring is partitioned into two logical token rings without any faults. The result is illustrated in Fig. 5(c).

With the token bus and its specific MAC protocols, the faults introduced in the etextiles only affect the nodes in failure state or the local piece of the bus. When the simultaneous faults are presented on the every ring as in Fig. 2(a) and (b), local full connectivity is still preserved. No remaining node will be abrupt by the faults as in the original token grid network. 


\section{Network Performance}

In this section, the performance of the EGNTB is described. We evaluate the approximate performance of maximum throughput and conduct simulation experiments to obtain the delay time with several faults.

Given that there is no fault on the network, the EGNTB is an original token grid network logically. Thus, the maximum capacity of the token grid network that is proposed by T.D. Todd in [10] can be used as the approximate throughput of the EGNTB. Let $\tau$ represent the node-to-node latency, token denote the token transmission time and $T$ is the transmission time of a data packet. $R$ presents the number of the rows. The approximate throughput of the square EGNTB with uniform load can be calculated as the following equation [10]:

$$
C=\frac{2 R(R+1)}{2 R+1} \cdot \frac{1}{1+t_{\text {token }} / T+\tau / T} .
$$

And to test the network performance with faults, we also conduct simulation experiments by using the physical layer model of the EGNTB on the Matlab. An EGNTB network of 16 nodes (4 Rows, 4 Columns) is studied on different fault occasions to obtain communication costs. An original e-textile token grid of 16 nodes is also tested under the same conditions and the results are used for comparison. Node $(1,1)$ is selected to send a data packet to every other node in the network. A data packet transmission comes to an end at the instant when the Node $(1,1)$ receives a reply from the destination node. Then a new transmission follows in the same way. Due to the virtual communicating bus we implement in the Matlab model, the time cost is recorded as a factor of a time unit. The time unit is defined as the delay that a token moves from a node to its direct successor on the token ring without any faults. The cost for transmitting fifteen data packets (fifteen destination node in the grid) is recorded.

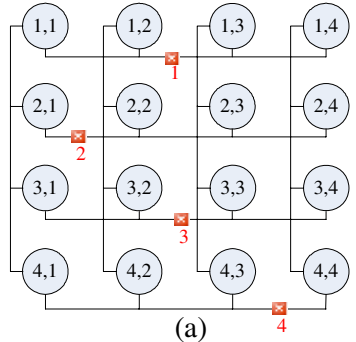

(a)

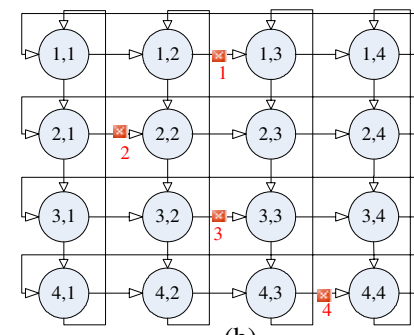

(b)

Fig. 6. Several faults introduced into the communication network: (a) EGNTB with detailed cases of faults (b) the original e-textile token grid with same link faults as in (a)

As shown in Fig. 6 (b) and (a), four cases of the link/bus faults are introduced into the communication networks. To conserve space, we only discuss the experiments on the link/bus faults. 
Table 1. Simulation results

\begin{tabular}{ccc}
\hline Case & EGNBT & $\begin{array}{c}\text { Original e-textile Token } \\
\text { Grid }\end{array}$ \\
\hline No Faults & 252 & 252 \\
One Fault of case 2/3/4 & 252 & 252 \\
The Fault of case 1 & 340 & 384 \\
One Fault of case 2/3/4 & 386 & 450 \\
$\quad$ and the fault 1 & & \\
$\quad$ (2 faults) & & \\
Two faults of case 2/3/4 & 430 & \\
and the fault 1 & & \\
$\quad$ (3 faults) & & Infinite value \\
All four faults & 476 &
\end{tabular}

It is noted that single fault of case 2 or 3 or 4 has no effect on the total cost of the delay time. The fault is tolerated by the grid topology of the network.

In the case of fault 1 , when a data packet is transmitted to a destination node on the Row 1, the original token grid with link fault 1 has to bypass the disconnected ring by a "Wrong Route". For example, if the destination of the data packet is node $(1,2)$, node $(1,1)$ routes the packet by two merge configurations at Node $(2,1)$ and Node $(4$, 2) respectively to turn around the link with fault 1 . For the EGNTB with the fault 1 , additional delay is observed in the two transmissions which have the destination nodes are node $(1,3)$ and node $(1,4)$ respectively. The RTB 1 is cut into two pieces and thus additional merge configuration is requested to route the data packet. Local connection of the two logical sub rings on row 1 is preserved. Meanwhile, the data packet transmission from node $(1,1)$ to node $(1,2)$ has less delay on the sub logical ring than that on the original proper logical ring. Because of the factors, the additional delay cost is less than that of the original token grid.

With the link fault 1 , the addition of the fault 2,3 or 4 to the networks has the same effect on the transmission from Node $(1,1)$ to the destination nodes not on the same columns and rows (nine such nodes in the Fig. 3(a) neither in Row 1 nor in Column 1). Some nodes are requested to be in merged configuration to fulfill the data transmission task. The more faults exit in the network, the more nodes with the merging configuration is requested. However, while a link fault can break out the communication on the row or column in the original e-textile token grid, the logical rings in EGNBT can tolerate some bus faults for the sub logical rings still connect the nodes locally.

In the presence of all the four link/bus faults, the original e-textile token grid is disabled and thus the time delay is an infinite value. The EGNTB can maintain the full network connectivity, but the delay cost is approximately two times that of the network without any faults. Table 1 lists the results obtained from the simulation experiments. These results show the EGNTB can improve the ability of e-textile applications to tolerate faults. On the occasions of same faults discussed above, the new network can provide the communication services of less delay time. Furthermore, the feature of a graceful variation of performance with the number of faults is also inherited. 


\section{Conclusions}

The harsh environment of e-textile applications requires a fault-tolerant communication scheme to reduce the time cost of faults or low power operations. In this paper, we introduce token buses to connect the nodes in e-textiles, instead of the single rings. Based on the specific protocol, the EGNTB implements the operations logically similar to the original token grid network. Thanks to the token bus, the new network inherits the high reliability of the bus topology and has the same approximate throughput with uniform load.

Simulation results show the new communication network proposed in this paper can enhance the ability of e-textile applications to tolerate faults. In the presence of same faults, the new network can provide the communication services of less delay time. And even when a fabric is torn into several parts, the full-connectivity in the local parts is also preserved in every fragments of the fabric. Furthermore, the EGNTB inherits the feature that the network performance degrades gracefully as the number of faults increases.

\section{References}

1. D. Marculescu, R. Marculescu, N. H. Zamora, P. Stanley-Marbell, P. K. Khosla, S. PARK, S. Jayaraman, S. Jung, C. Lauterbach, W. Weber, T. Kirsein, D. Cottet, J. Grzyb, G. TrÖster, M. Jones, T. Martin, and Z. Nakad, "Electronic Textiles: A Platform for Pervasive Computing", Proceedings of the IEEE, VOL. 91, NO. 12, 1995-2018, December 2003.

2. M. Jones, T. Martin, Z. Nakad, R. Shenoy, T. Sheikh, D. Lehn, and J. Edmison, "Analyzing the Use of E-textiles to Improve Application Performance", IEEE Vehicular Technology Conference 2003, Symposium on Wireless Ad hoc, Sensor, and Wearable Networks (VTC 2003)(extended abstract), October 2003.

3. Tanwir Sheikh, Modeling of Power Consumption and Fault Tolerance for Electronic Textiles, Bradley Department of Electrical and Computing Engineering, Virginia Tech, September 2003.

4. Zahi Nakad, Architecture for e-Textiles. PhD thesis, Bradley Department of Electrical and Computing Engineering, Virginia Tech, 2003.

5. The Georgia Tech wearable motherboard: The intelligent garment for the 21st century (1998). [Online]. Available:http://www.smartshirt.gatech.edu.

6. T. D. Todd, "The Token Grid: Multidimensional Media Access for Local and Metropolitan Networks", Proceedings of the eleventh Annual Joint Conference of the IEEE Computer and Communications Societies, pp. 2415-2424, 1992.

7. Z. Nakad, M. Jones, and T. Martin, "Communications in Electronic Textile Systems", Proceedings of the 2003 International Conference on Communications in Computing, pp. 3743, June 2003.

8. Z. Nakad, Mark Jones, and Thomas Martin, "Fault-Tolerant Networks for Electronic Textiles", Proceedings of the 2004 International Conference on Communications in Computing, Las Vegas, June 2004.

9. F. E. Ross, "An Overview of FDDI: The Fiber Distributed Data Interface", IEEE J. Select. Areas Commun., vol. 7, pp. 1043-1051, Sept. 1989.

10. T. D. Todd, "The Token Grid Network", IEEE/ACM Transactions on Networking, vol. 2, No. 3, pp. 279-287, June, 1994. 